\title{
Increased Speed: 3D Silicon Sensors; Fast Current Amplifiers
}

\author{
Sherwood Parker, Angela Kok, Christopher Kenney, Pierre Jarron, Jasmine Hasi, Matthieu Despeisse, \\ Cinzia Da Via, and Giovanni Anelli
}

\begin{abstract}
The authors describe techniques to make fast, subnanosecond time resolution solid-state detector systems using sensors with 3D electrodes, current amplifiers, constant-fraction comparators or fast wave-form recorders, and some of the next steps to reach still faster results.
\end{abstract}

Index Terms-Fast pulses, short time resolution, silicon detectors, solid-state detectors, speed, 3D sensors.

\section{INTRODUCTION: A BRIEF History OF SHORT TIMES}

A LTHOUGH reasonably fast readout electronics was available [1] in actual use, silicon radiation sensors, when originally introduced, were not particularly fast compared with contemporaneous detectors. For example, for the silicon detectors used in the pioneering UA2 experiment at CERN, "the width of the shaped signal is $2 \mu \mathrm{s}$ at half amplitude and $4 \mu$ s at the base" [2]. Individual sensor elements were generally relatively large, and so had high capacitance while the charge from them, about 15 000-25 000 electrons and with no amplifying elements in the sensors, meant the voltage signals were small. So slow amplifiers, which integrated out a large part of the white noise generated at the input, were used.

The development of microstrip sensors [3] greatly reduced the capacitance between the top and bottom electrodes, adding a smaller, but significant one between adjacent strips. The first custom, 128-channel, VLSI readout chip [4], [5], developed for those microstrip detectors and which made their use practical for collider vertex detectors, had amplifiers with 20-25 ns rise times. (They were set by the need to roll off amplification below the frequency where one half-cycle was equal to the round trip

Manuscript received April 23, 2010; revised August 18, 2010, October 27, 2010; accepted December 12, 2010. Date of publication March 17, 2011; date of current version April 13, 2011. This work was supported in part by the U.S. Department of Energy under Grants DE-FG02-04ER41291, and was performed in part at the Stanford Nanofabrication Facility (a member of the National Nanotechnology Infrastructure Network), which is supported by the National Science Foundation under Grant ECS-9731293.

S. Parker is with the University of Hawaii, c/o Lawrence Berkeley Laboratory, Berkeley CA 94720 USA (e-mail: sher@slac.stanford.edu).

A. Kok is with SINTEF, MinaLab, Blindern 0314 Oslo, Norway.

C. Kenney and J. Hasi are with the SLAC National Accelerator Laboratory, Menlo Park, CA 94025-7090 USA.

P. Jarron and G. Anelli are with CERN, 1211 Genève 23, Switzerland.

M. Despeisse is with the Ecole Polytechnique Fédérale de Lausanne (EPFL), Institute of Microengineering (IMT), Photovoltaics and Thin Film Electronics Laboratory, CH-2000 Neuchatel, Switzerland.

C. Da Vià is with the Physics and Astronomy Department, University of Manchester, MI3 9PL Manchester, U.K. (e-mail: cinzia.da.via@cern.ch).

Color versions of one or more of the figures in this paper are available online at http://ieeexplore.ieee.org.

Digital Object Identifier 10.1109/TNS.2011.2105889 time for the signal to go through the inverter and be fed back to the input. Otherwise the positive feedback would have produced a chip with 128 oscillators and no amplifiers.)

The planned use of microstrip detector arrays at colliders with short inter-collision times required a further increase in speed. Reference [6] describes a current amplifier with a rise-time of $4 \mathrm{~ns}$ and a pulse width at the base of $30 \mathrm{~ns}$. All of these early amplifiers were developed to read out planar sensors.

Two developments now allow higher speeds in timing using silicon detector systems:

- Silicon sensors with 3D $\mathrm{n}^{+}$and $\mathrm{p}^{+}$doped silicon electrodes that penetrate through the silicon bulk [7]-[26] allow charge from long tracks to be collected in a rapid, smooth high-current burst. The electrodes are formed by etching holes and diffusing dopant gasses into the surrounding single-crystal silicon. It is expected that the electrodes of the fastest sensors will be flat planes spanning both the thickness and much of the sensor width. They can form high, near-uniform fields and will be discussed in Section VII. To survive the fabrication steps with a reasonable yield, the electrode holes of such sensors must be filled with polycrystalline silicon. Fig. 1 shows a simplified view of a more common electrode array which may have but does not require such a filling [27]-[29] and compares collection paths of 3D and planar sensors.

- Continued developments in integrated circuit technology fabrication permit the design and fabrication of even higher speed current amplifiers [30]-[32]. They can produce large voltage signals from those high speed input currents. Up to the sensor speed, such signals grow more rapidly than white noise with increasing bandwidth. In parallel, extensive work is in progress developing fast circuits for timing photo-detector signals from light sources such as Cerenkov counters [33], [34] some of which might be applied to silicon sensor readout.

One possible use of timing resolution, if it could be made fast enough, would be in small-angle, far-forward detectors at colliders where relative timing of the two scattered particles could locate their vertex position among several possible vertices. The arrangement would be more like that at fixed-target experiments than at colliders and could allow for multiple, small-area detectors run at a lower temperature than that of the central detectors.

First we examine some sources of timing errors in Section II, look at several of them in detail in Sections III-VI, cover general methods of reducing them in Section VII, and look at what our data indicates about the main remaining error source for 3D detectors: noise in Section VIII. The general physical idea of these 


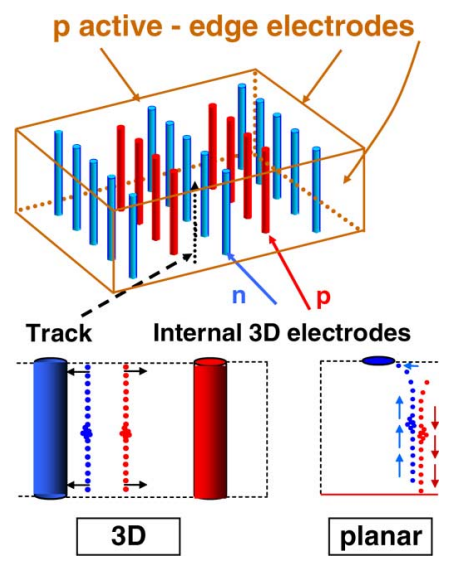

Fig. 1. (top) Schematic diagram of 3D sensor with normally-incident track. (bottom) Schematic detail of tracks with an ionization cluster. Electrons drift to the left in the 3D sensor in the left diagram and up in the planar diagram on the right.

two methods is explained in a condensed form in Section IX. Detailed steps for calculations of timing errors due to noise are given in Section X, the results are given in Section XI, possible future work in XII and conclusions in XIII.

\section{TIMING ERRORS}

Neglecting effects of radiation damage which will add additional items to the list below such as charge capture and increased drift speeds, the difference between the arrival time of an incoming particle and the value measured for that time can come from a number of sources.

1. Variations in track location

2. Variations in track direction-1 and $\mathbf{2}$ can affect the shape and timing of the detected pulse.

3. Variations in total ionization signal- Depending on the electronics, this can affect the trigger time.

4. Variations in ionization location along the track-Delta rays-high energy, but still generally non-relativistic, and so heavily-ionizing ("knock-on") electrons, found at varying positions along the track. They make a charge cluster which gives a signal proportional to the dot product of their charge times collection drift velocity and the Ramo weighting field [35]-[37]. The current signal induced by the cluster of delta ray charges drops to zero over a short period of time as the cluster reaches the electrode and the charge velocities drop to zero. In planar detectors, this happens at random times during the pulse, limiting the accuracy of getting a unique time from the pulse.

5. Diffusion of charge carriers-This causes a spreading in space of the drifting ionization charges which can modify the development of the signals induced by the charges.

6. Magnetic field effects affecting charge collection- $E \times$ $B$ forces may shift the collection paths.

7. Measurement errors due to noise- This is currently the major error source.

8. Incomplete use of, or gathering of, available information-This is a challenge mainly for the data acquisition electronics which, for high speed, will often have to face power and heat removal limitations. Except for the use of the pulse shape, this topic is not covered in this paper.

A sensor with parallel 3D electrodes made from trenches filled with doped polycrystalline silicon separated by $100 \mu \mathrm{m}$, and described in Section VII, can be made to be insensitive to the errors 1-6 above. In addition, one possible initial use would be timing of normally-incident particles with known locations and not in a magnetic field, making 1, 2, and 6 unimportant. In the barrel (but not end-cap) layers of detectors such as ATLAS, CMS, and CDF with solenoidal magnetic fields, 3D sensors will have parallel $\mathrm{E}$ and $\mathrm{B}$ fields or with $\mathrm{E} \times \mathrm{B}$ in the direction of the track. This will at most just shift the ionization column along its own length, producing no change in the collection path, so again 6 is unimportant.

However, additional discussion and calculations must be made for 3, 4, 5, and 7-Landau fluctuations and the related delta rays, diffusion, and noise-to evaluate their effects.

\section{DeLta RAYS}

To understand the effect of delta rays on timing, we must calculate the number produced per unit track length, their energy distribution, their typical ranges, and their initial angle [38], [45]. Then we use Casino V2.42, a Monte Carlo program originally written for scanning electron microscopy, to see how the delta rays propagate [39].

Delta rays are largely responsible for Landau fluctuations in $\mathrm{dE} / \mathrm{dx}$ as well as being a potentially serious source of timing errors in planar sensors. Formulas for their maximum energy, number per unit track length as a function of delta ray kinetic energy, and their production angle can be found in [38], [45] equations (27.2, 5, and 6). The delta ray upper energy limit, $\mathrm{T}_{2}$ from (27.2) is normally far above any found on a typical track. For either incident multi-GeV pions or for $5 \mathrm{TeV}$ protons $(\beta \approx$ $1,15<\gamma<5000), \mathrm{T}_{2}$ can range from several hundred $\mathrm{MeV}$ to close to $5 \mathrm{TeV}$, while typical delta ray kinetic energies are in the $\mathrm{keV}$ region, and their ranges that are quite short compared with silicon sensor thicknesses.

Integrating the equation, (27.5) from $\mathrm{T}=\mathrm{T}_{1}$ to $\mathrm{T}_{2}$ for a silicon sensor thickness of $s \mathrm{~g} / \mathrm{cm}^{2}$, using $Z / A$ for silicon $=0.498$, density $=\rho_{\mathrm{Si}}=2.329 \mathrm{~g} / \mathrm{cm}^{3}$, incident particle (charge/qe) $=z=1$, incident particle (velocity $/ \mathrm{c})=\beta \approx 1$, and setting the spin-dependent term $\mathrm{F}(\mathrm{T})$ (which is also close to 1 ) equal to 1 , we find $n$, the mean number of delta rays produced per thickness $d$ between kinetic energies $T_{1}$ and $T_{2}$ is

$$
n=76.5 d \int_{T_{1}}^{T_{2}} \frac{d T}{T^{2}}=76.5 d\left(\frac{1}{T_{1}}-\frac{1}{T_{2}}\right)=\frac{3.15 \mathrm{keV}}{T_{1}}
$$

where, in the last part, we have neglected $1 / T_{2}$ and used $d=$ $0.0396 \mathrm{gm} / \mathrm{cm}^{2}$, the value for the $177 \mu \mathrm{m}$ thick sensors used to collect the data of Sections X-XI. Their production angles relative to the track direction, starting with the very probable $T_{1}=3 \mathrm{keV}$, and continuing with the increasingly less probable $T_{1}=10,30$, and $60 \mathrm{keV}$, are $86^{\circ}, 8^{\circ}, 8^{\circ}$, and $76^{\circ}$. 

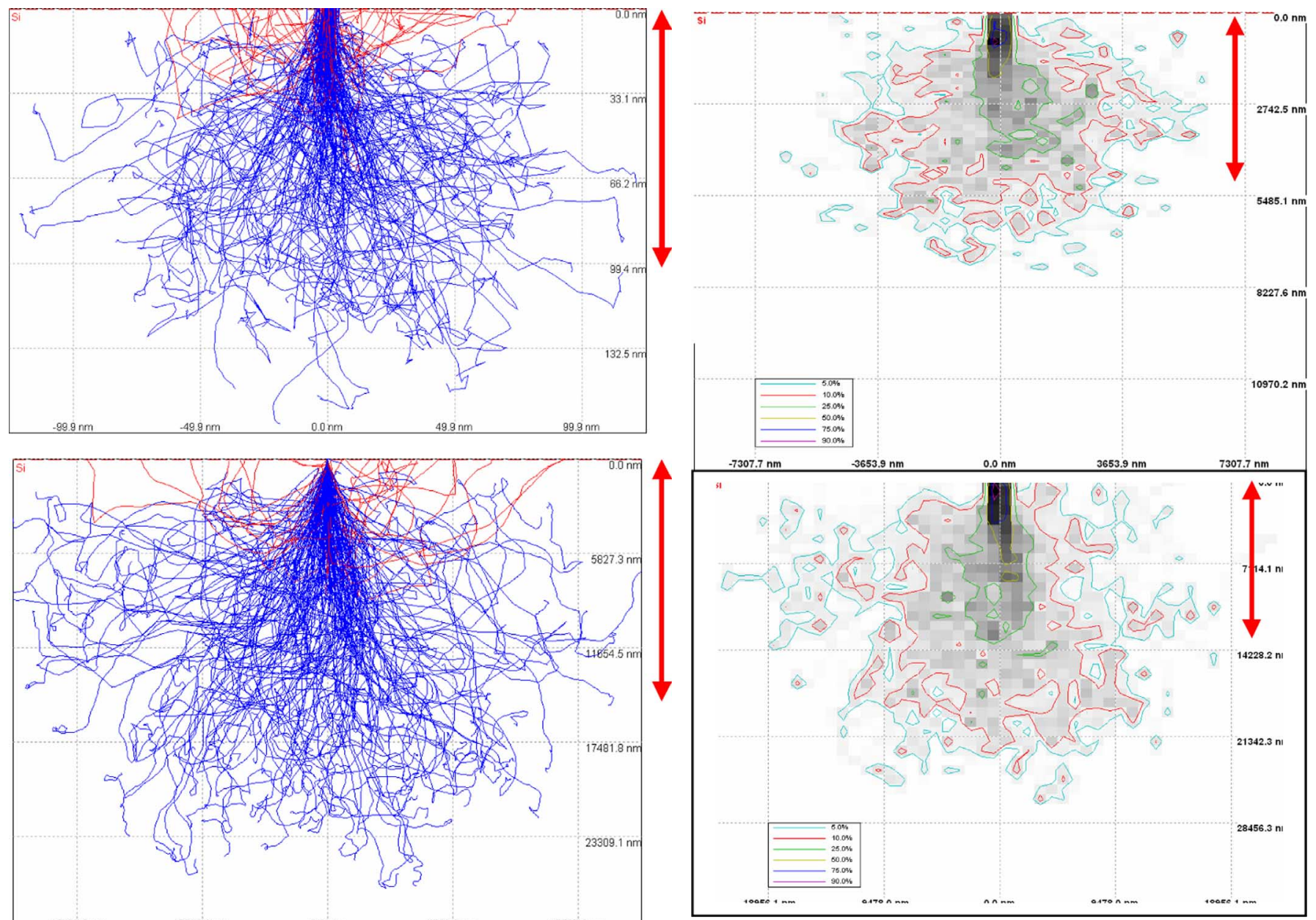

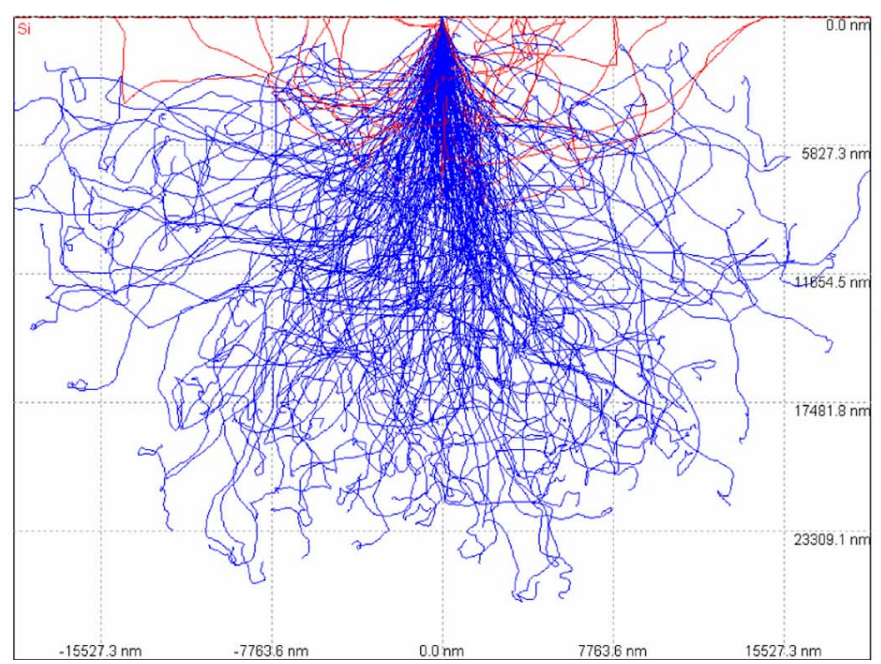

Fig. 2. (top) $2003-\mathrm{keV}$ delta rays. Red lines in the electronic copy trace paths of backscattered tracks. The arrow is $0.1 \mu \mathrm{m}$ long. Arrows for similar plots of 10 and 30-keV delta rays are 1 and $5 \mu \mathrm{m}$ long. (bottom) $20060-\mathrm{keV}$ delta rays. Red lines in the electronic copy trace paths of backscattered tracks. The arrow is $15 \mu \mathrm{m}$ long.

It may be useful to compare these energies with the minimum of the mean energy loss given by the Bethe-Bloch equation, $d E / d x_{\min }=1664 \mathrm{keV}$ per $\mathrm{gm} / \mathrm{cm}^{2}$ for silicon, which will give a mean total energy loss in the $177 \mu \mathrm{m}$-thick silicon sensor of $\Delta T=2.329 \mathrm{gm} / \mathrm{cm}^{3} \times 0.0177 \mathrm{~cm} \times 1664 \mathrm{keV}$ per $\mathrm{gm} / \mathrm{cm}^{2}=68.6 \mathrm{keV}$.

Typical paths and energy deposition for 3-60 keV delta rays, given by Casino, are shown in Fig. 2 and Fig. 3. As can be seen in Fig. 2, the common $3 \mathrm{keV}$ delta rays with typical ranges of $0.1 \mu \mathrm{m}$ leave their ionization so close to the track that they do not influence the timing for tracks parallel to the electrodes of $3 \mathrm{D}$ sensors.

$10 \mathrm{keV}$ delta rays have typical ranges of $0.5 \mu \mathrm{m}$. They or higher energy delta rays are present on $30 \%$ of the traversals. With no preferential orientation in the plane perpendicular to the track they will be oriented to lead the rest of the track about $1 / 4$ of that $30 \%$. With typical electron drift velocities of about $5 \times 10^{6} \mathrm{~cm} / \mathrm{sec}$, the leading edge of the delta ray could reach an n-type electrode up to $10 \mathrm{ps}$ ahead of the main track, ending that part of the pulse a bit early. Delta rays with lateral orientations
Fig. 3. Energy deposition coutours of 200 delta rays. (top) $30 \mathrm{keV}$. The $50 \%$ containment contour goes to a depth of $2.0 \mu \mathrm{m}$ with a maximum full width of $0.8 \mu \mathrm{m}$. The $75 \%$ contour goes to a depth of $4.3 \mu \mathrm{m}$ with a maximum full width of $2.7 \mu \mathrm{m}$. The arrow is $5 \mu \mathrm{m}$ long. (bottom) $60 \mathrm{keV}$. The $50 \%$ containment contour goes to a depth of $8.0 \mu \mathrm{m}$ with a maximum full width of $2.0 \mu \mathrm{m}$. The $75 \%$ contour goes to a depth of $13.5 \mu \mathrm{m}$ with a maximum full width of $7.3 \mu \mathrm{m}$. The arrow is $15 \mu \mathrm{m}$ long.

would just increase the signal amplitude and so the signal to noise ratio. With typical deposition energies for the entire track of $60-80 \mathrm{keV}$ and typical signal-to-noise ratios of 15 to 1 or better, $10 \mathrm{keV}$ is at the start of the range where the presence of such delta rays could matter.

Several detectors in sequence would not only provide improved statistical accuracy, but could allow relative weighting variations for the traversals of the track. The most accurate detectors-ones using fast waveform recorders-might, from pulse shape, recognize and reduce the weighting of data with delta-ray induced errors and increase weighting where the delta rays just increase the signal. This will be covered in Section VIII.

Continuing up the energy scale, $30-60 \mathrm{keV}$ delta rays have typical ranges of $5-15 \mu \mathrm{m}$, with $50 \%$ ionization containment contours less than half of that (see Fig. 3). They could affect timing, but can be identified by the large increase in pulse height, which will place them in the Landau tail. 

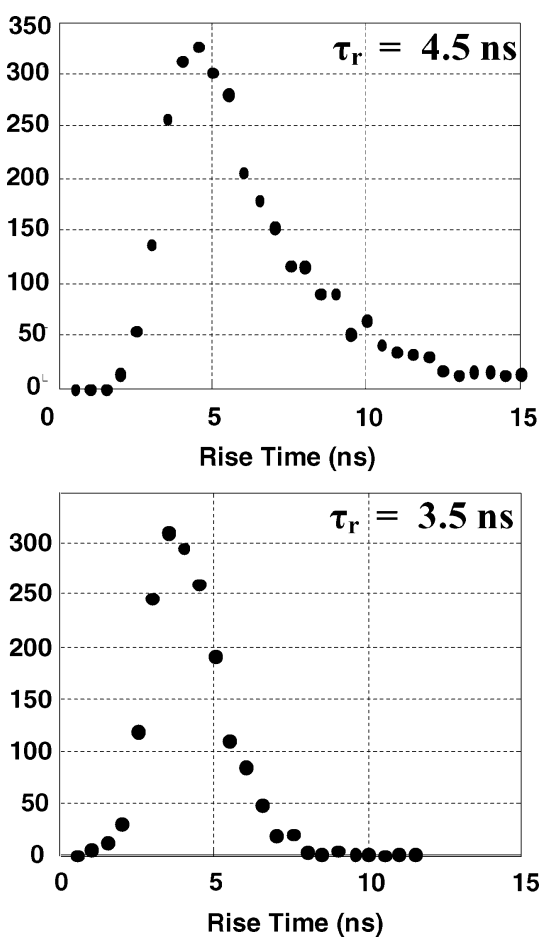

Fig. 4. Rise time distributions in the hex sensor of Fig. 8 for $35 \mathrm{KeV}$ X-rays from a ${ }^{133} \mathrm{Ba}$ source (top) and beta rays from ${ }^{90} \mathrm{Sr}$ (bottom). The un-collimated, penetrating ${ }^{90} \mathrm{Sr}$ beta rays deposit a broad range of ionization energies with the most probable about $35 \mathrm{keV}$ but with a long Landau tail. The vertical axes give the number of events per bin. The full widths at half max (10 and $9.5 \mathrm{~ns})$ and fall times (5.5 and $4 \mathrm{~ns}$ ) were also longer for ${ }^{133} \mathrm{Ba}$.

\section{EfFects of Charge Clusters on Charge Collection: DELTA RAYS AND X-RAYS}

Although the primary purpose of this paper is to study factors leading to improved timing of high-energy particle tracks, some of the initial data was taken with a ${ }^{133} \mathrm{Ba} \mathrm{K}$-capture source where the resulting ${ }^{133} \mathrm{Cs}$ emits most frequently a 35 $\mathrm{keV} \mathrm{X}$-ray. Although the amplifier had not yet been set for the highest speeds, and the overshoot was larger, the $\mathrm{X}$-rays were seen to have distinctly slower speeds than the betas from ${ }^{90} \mathrm{Sr}$ taken just before or after and with the same settings.

This was surprising, since the X-rays did not have the large collection-distance variations of the betas. Fig. 4 shows the number of events per bin for the two rise time distributions. The differences were obvious, just looking at the oscilloscope traces during data collection. The ionization clusters made by the $\mathrm{X}$-rays are not that different from those made by high-energy delta rays, and so it is important to understand these slower $\mathrm{X}$-ray pulses.

An exact prediction of this effect would require the Monte Carlo generation of tracks, the calculation of the ionization charge diffusion, the drift under the bias and ionization-charge fields and the dot product of the resultant current with the Ramo weighting field. We can use the results of the calculations in Section III which should hold for the delta ray tracks to make a rough estimate of the attraction of the hole and electron clouds as they separate and compare this with the bias field that is causing them to separate. The bias field is of the order of $\mathrm{E}_{\text {bias }} \approx 20 \mathrm{~V} / 50 \mu \mathrm{m}=4 \times 10^{5} \mathrm{~V} / \mathrm{m}$. The hole-electron attractive fields should be largest as the holes and electron distributions have just about pulled apart but are still next to each other. This separation distance is very approximately equal to the arrow lengths in the delta ray figures. We will use: $0.1,1.0,5.0$, and $15 \mu \mathrm{m}$ for $3,10,30$, and $60 \mathrm{keV}$

from Fig. 2 with the charge in each cluster as

qelectron $\times \mathrm{K}$ (energy of the X-ray in $\mathrm{eV}) / 3.62 \mathrm{eV}$

and grouping these charges at the separation distances above for $3,10,30$, and $60 \mathrm{keV}$, we get

$\mathrm{E}$ (holes to electrons or vice versa) $\approx 1.0 \times 10^{7}, 3.3 \times 10^{5}$, $4.0 \times 10^{4}$, and $8.9 \times 10^{3} \mathrm{~V} / \mathrm{m}$.

The large attractive fields at low energy extend over a shorter distance, but all at about $30 \mathrm{keV}$ and below look like they could have a significant effect on collection times. A similar effect should affect the very end of the pulse for minimum-ionizing particles in a trench-electrode detector, as it will delay the delta rays, tending to keep them behind the main track, but should not make a significant difference during the main drift time.

\section{DIFFUSION}

As any charge travels to an electrode, it diffuses around the point on the path it would otherwise follow having a Gaussian distribution with:

$$
\sigma=\sqrt{2 D t}=\sqrt{2 D \frac{s}{\beta c}}
$$

where $\mathrm{D}$ is the diffusion constant, often given as $35 \mathrm{~cm}^{2} / \mathrm{s}$ for electrons and $12 \mathrm{~cm}^{2} / \mathrm{s}$ for holes, $s$ is the drift distance and $\beta c$ the velocity. This will cause a spread in arrival times, given in Table I, of

$$
\sigma_{t}=\sqrt{2 D \frac{s}{\beta^{3} c^{3}}} .
$$

However, D can vary from these values, depending on the value of the electric field, $\mathbf{E}$, the temperature, the direction-parallel or perpendicular - to $\mathbf{E}$, and to a small extent, neglected here, the direction of the crystal axis [40], [41]. Table I shows some of the important values derived from [40] and [41].

\section{SPEED AND TRIGgERS From RANDOM NOISE}

It is often assumed that limiting the band width will reduce noise, and so reduce false triggers from noise. However, the fast intrinsic pulse times, $\tau_{\text {det }}$, can, with well-designed current amplifiers, produce high signal-to-noise ratios. The output signal current is proportional to the detector speed (which depends on the speed of the incoming ionization charge and the amplifier speed), while, for instance, noise across a resistance such as a transistor channel only grows as the square root of the frequency spread. So increasing the speed of an amplifier up to the highest predominant speeds of the sensor can then improve the signal-to-noise ratio of a current-amplifier based circuit. 
TABLE I

VELOCITIES, DiFFUSION, AND COLLECTION TIMES FOR DRIFT ACROSS A 100 $\mu \mathrm{M}$ Parallel Plate TRENCh Electrode GaP

\begin{tabular}{|c|c|c|c|c|c|}
\hline \multirow[b]{2}{*}{ temperature } & \multicolumn{2}{|c|}{ electrons } & \multicolumn{2}{|l|}{ holes } & \multirow{2}{*}{$\begin{array}{c}\text { units } \\
{ }^{\circ} \mathbf{K}\end{array}$} \\
\hline & 293.15 & 245 & 293.15 & 245 & \\
\hline $\mathrm{V}(\mathrm{E}=0.2 \mathrm{~V} / \mu \mathrm{m})$ & 2.6143 & 4.0 & 0.92035 & 1.41 & $\mathrm{~cm} / \mu \mathrm{s}$ \\
\hline $\mathrm{V}(\mathrm{E}=0.5 \mathrm{~V} / \boldsymbol{\mu m})$ & 4.9286 & 7.0 & 2.0657 & 2.22 & $\mathrm{~cm} / \mu \mathrm{s}$ \\
\hline $\mathrm{V}(\mathrm{E}=1.0 \mathrm{~V} / \boldsymbol{\mu m})$ & 6.9072 & 8.8 & \begin{tabular}{|l|}
3.4598 \\
\end{tabular} & 4.62 & $\mathrm{~cm} / \mu \mathrm{s}$ \\
\hline $\mathrm{D}(0.2 \mathrm{~V} / \mu \mathrm{m})$ & 32 & & 12.3 & & $\mathrm{~cm}^{2} / \mathrm{s}$ \\
\hline $\mathrm{D}(0.5 \mathrm{~V} / \mu \mathrm{m})$ & 21 & & 10.7 & & $\mathrm{~cm}^{2} / \mathrm{s}$ \\
\hline $\mathrm{D}(1.0 \mathrm{~V} / \mu \mathrm{m})$ & 13.5 & & 7.36 & & $\mathrm{~cm}^{2} / \mathrm{s}$ \\
\hline $\mathrm{t}(\mathrm{E}=0.2 \mathrm{~V} / \mu \mathrm{m})$ & 3.83 & 2.83 & 10.9 & 7.57 & ns \\
\hline$\sigma_{t, \text { diffusion }}$ & 0.19 & & 0.56 & & ns \\
\hline $\mathrm{t}(\mathrm{E}=\mathbf{0 . 5 \mathrm { V } / \boldsymbol { \mu } \mathrm { m } )}$ & 2.03 & 1.61 & 4.84 & 3.53 & ns \\
\hline$\sigma_{t, \text { diffusion }}$ & 0.059 & & 0.16 & & ns \\
\hline$t(E=1.0 V / \mu m)$ & 1.45 & 1.21 & 2.89 & 2.22 & ns \\
\hline$\sigma_{t, \text { diffusion }}$ & 0.029 & & 0.06 & & ns \\
\hline \multicolumn{6}{|c|}{$\max \delta$-ray collection times for $E=1 \mathrm{~V} / \mu \mathrm{m}$} \\
\hline $3 \mathrm{keV}$ & 1.9 & 1.5 & 3.8 & 2.8 & ps \\
\hline $10 \mathrm{keV}$ & 14 & 11 & 29 & 22 & ps \\
\hline $30 \mathrm{keV}$ & 101 & 80 & 202 & 152 & ps \\
\hline $60 \mathrm{keV}$ & 362 & 284 & 723 & 541 & ps \\
\hline \multicolumn{6}{|c|}{$V=$ drift velocity, $D=$ diffusion coefficient parallel to $V$} \\
\hline
\end{tabular}

One example, useful for studying the rate of false noise triggers, is the number of traversals per second, $n$, of a Gaussian noise current $I_{n}$ with a positive slope past a threshold level, $I_{t}$, [42]

$$
n=\left[\frac{\int_{0}^{\infty} \nu^{2} p(\nu) d \nu}{\int_{0}^{\infty} p(\nu) d \nu}\right]^{0.5} \exp \left(\frac{-I_{t}^{2}}{2 \overline{I_{n}^{2}}}\right) .
$$

Here $p(\nu)$ is a frequency-dependent filter or transmission factor. If it is flat from $\nu 1$ to $\nu 2$, and with (typically) $\nu_{2} \gg \nu_{1}$, we have

$$
n=\left[\frac{\nu_{2}^{3}-\nu_{1}^{3}}{3\left(\nu_{2}-\nu_{1}\right)}\right]^{1 / 2} \exp \left(\frac{-I_{t}^{2}}{2 \overline{I_{n}^{2}}}\right)=\frac{\nu_{2}}{\sqrt{3}} \exp \left(\frac{-I_{t}^{2}}{2 \overline{I_{n}^{2}}}\right)
$$

$I_{t}$ can be set equal to a fraction $a_{1}$ of $\overline{I_{\text {det }}}$, the average value of the signal pulse current

$$
I_{t}^{2}=a_{1}^{2} \overline{I_{\operatorname{det}}^{2}}=a_{1}^{2}\left\langle\frac{q}{\tau_{\operatorname{det}}}\right\rangle^{2}=a_{2} \nu_{\operatorname{det}}^{2} .
$$

Here $q$ is the total charge, $\tau_{\text {det }}$ is the pulse duration, and $\nu_{\text {det }}$ is $1 / \tau_{\text {det }}$. The constants $a_{1}$ and $a_{2}$ are independent, to first order, of $\nu$. White noise currents in electronics capable of matching detector speeds, but not introducing unnecessary noise, will have an upper frequency limit, $\nu_{h} \approx \nu_{\mathrm{det}} \approx \nu_{2}$ (in (5) above) and

$$
\overline{I_{n}^{2}}=a_{3} \Delta \nu=a_{3}\left(\nu_{h}-\nu_{l}\right) \approx a_{3} \nu_{h}=a_{4} \nu_{\mathrm{det}}
$$

where $\nu_{l}$ is the lower frequency limit. (For a resistor, $a_{3}$ would equal $4 \mathrm{kT} / \mathrm{R}$.) The threshold level squared can be increased in proportion to the detector speed squared, giving a near-exponential drop in noise counts that is proportional to the detector speed

$$
n=\left(\frac{\nu_{\mathrm{det}}}{\sqrt{3}}\right) \exp \left(-\frac{a_{2} \nu_{\mathrm{det}}^{2}}{2 a_{4} \nu_{\mathrm{det}}}\right)=\left(\frac{\nu_{\mathrm{det}}}{\sqrt{3}}\right) \exp \left(-a_{5} \nu_{\mathrm{det}}\right) .
$$

A simple integrator will not benefit from this speed. The circuit must respond only to rapid changes in voltage on an integrating capacitor or to high instantaneous currents in a resistive circuit.

\section{REDUCING TIMING ERRORS}

At some level, systems can be chosen that will permit corrections to the effects listed in Section II. Multiple layers can provide the track information for 1 and 2, the ability to take advantage of larger signals from Landau fluctuations and to average noise, help with 3 and 7 . Low temperatures will decrease noise and increase circuit speeds.

For several reasons, 3D sensor signals can be intrinsically faster than planar ones.

- The 3D electrode spacing can be less than the wafer thickness, so carrier drift distances are shorter.

- Depletion voltages are low so the use of over-depletion voltages means that collection fields can be more uniform and uniformly high.

- In addition, in 3D, the field lines end on surfaces which normally have more area than corresponding planar electrodes, so the ratio of the average drift fields to the peak field can be larger for 3D sensors (there is a price for this: increased electrode capacitance).

- Common, low energy delta rays, with a range short compared to the gap are collected, together with the adjacent track ionization, during a few picoseconds. If the track is parallel to the electrode, everything is collected during those few picoseconds at the end of the current pulse. Before this time, they are drifting close to the track, and give the same induced signals. Except for noise, the pulse will be very smooth, unlike those in planar sensors where the delta ray ionization is absorbed at random times during the pulse. Higher energy delta rays, with longer ranges, are rare and recognizable. Diffusion from (2) of about $2.6 \mu \mathrm{m}$ for electrons and $1.5 \mu \mathrm{m}$ for holes in the first ns and growing as the square root of time, modifies these signal shapes only slightly.

- Both types of 3D electrodes can be contacted on a single surface, so if capacitative readout for at least one type of electrode can be used, pulses from both the n-type and 


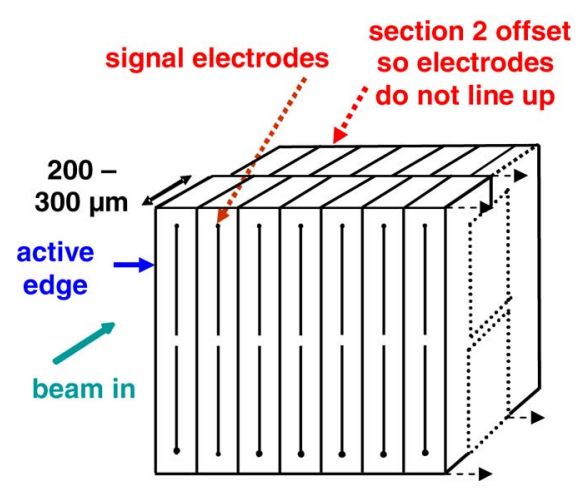

Fig. 5. Schematic diagram of part of one section of two of the planes in an active-edge 3D trench-electrode detector. Other offsets between the front and back sections besides the $1 / 2$ section one shown may also be used. For example a 3 section system might use offsets of $0,1 / 3,2 / 3$.

p-type electrodes may be recorded. This may improve both time and spatial resolution. The same magnitude of charge goes to spatially separated electrodes, providing additional track information and can improve the signal-to-noise ratio, giving better timing.

Trench (or "wall") electrodes (see Figs. 5 and 6 and [12]) provide additional advantages, particularly for tracks that are not close to electrodes or their subdivisions. A signal electrode having two $100 \mu \mathrm{m}$ gaps on both sides and a $250 \mu \mathrm{m}$ thickness (in the track direction) will have a capacitance of $0.527 \mathrm{pF}$ per mm of width.

- There are no null-field regions in the depleted substrate.

- They have a particularly high average field/peak field ratio.

- Far enough beyond the gap between adjacent p-p or n-n signal electrodes-perhaps several gap-lengths-where the field lines are essentially straight, the Ramo weighting field will also be nearly constant. Of course, short gaps will increase the capacitance.

- Larger p-n electrode gaps will lower the capacitance, improving the signal-to-noise ratio, and reduce the fraction of tracks crossing close to an electrode where analysis is more difficult. But they will require higher bias voltages and will have a longer collection time.

- Offsets for additional sections (one is shown in Fig. 5) are used so all tracks have at least part of their traversals away from the electrodes. (This prevents the case of a traversal of a track that is always near an n-electrode where the Ramo signal would be formed only by slower-moving holes.)

- If the bias electrodes are also instrumented, they will have the same magnitude, but opposite sense, current signals. The bias electrodes can then have pixel boundaries at the mid-points of the signal electrodes, roughly doubling the spatial resolution in the vertical direction in Fig. 5. This always provides a nearly constant Ramo field on at least one electrode plane.

- For moderate to high bias voltage levels $(\sim 50 \mathrm{~V})$ and low dopant levels $\left(\sim 5 \times 10^{11} / \mathrm{cm}^{3}\right)$ the depletion voltage of $\sim 2 \mathrm{~V}$ will introduce only a small change in the constant charge-carrier drift velocity. After irradiation, the electric field will not be uniform, but the velocity will be faster as the bias voltage can normally be raised, and at high- field values and velocity saturation, will again be nearly constant.

- The wall-to-wall pitch will normally be large compared to most all delta ray lateral ranges, so the ionization columns will move without shape changes, other than those due to diffusion, for all but a small subset of tracks crossing close to an electrode.

- There will be a varying delay until the electromagnetic pulse from the separating holes and electrons to travels to the electrodes. This delay is normally under a picosecond, given that $\mathrm{v}_{\mathrm{em}}=\mathrm{c} / \mathrm{n}$ and the index of refraction of silicon, $\mathrm{n}$, is only 3.95 [43].

- Beyond that delay, the time, shape, and magnitude of the leading edge of the pulse will depend mainly on the time of the track traversal, on the local drift velocity, and on the track location for tracks near pixel boundaries. Fig. 7 shows an idealized diagram of this expected induced current shape.

- With a known sensor signal input shape for tracks that are distant from pixel boundaries, use of a waveform recorder as an electronics input device-if they can be made to fit in the area of one pixel-would provide a powerful tool. Shifting the relative times of the known, noise-free pulse and the experimental waveform to find the time of best match would provide, from the noise-free pulse time, a measurement with reduced sensitivity to small-scale noise fluctuations.

- The dominant remaining source of error is noise. The next section describes an example of the improvement from the use of a waveform tracer and sample measurements of the size of this source of error.

Table I gives the maximum electron and hole drift times for a $100 \mu \mathrm{m}$ gap for tracks near one or the other trench electrode. It also gives the room temperature arrival time spread caused by diffusion-about $2 \%$ to $5 \%$ of the corresponding drift times. Even this small value should not significantly increase timing errors, as unlike delta rays, which may or may not be present, the particle statistics shaping this leading edge are high.

\section{NoISE AND TIME RESOLUTION}

Our trench-electrode sensor (see Fig. 6) was too small to give an adequate rate from our ${ }^{90} \mathrm{Sr}$ beta source, so we started with a $177-\mu \mathrm{m}$ thick 3D active-edge sensor having hexagonal cells with $50 \mu \mathrm{m}$-long hexagon sides, giving a maximum drift distance shorter than that of even our radiation-hard ATLAS sensors [26] (see Fig. 8). It had 16 sets of 20 hexagons with ganged central electrodes with $20 \mathrm{~V}$ bias at room temperature and was exposed to un-collimated ${ }^{90} \mathrm{Sr}$ betas. Each of the 16 columns went to one channel of a fast $0.13 \mu$ m-technology VLSI current-amplifier chip developed by M. Despiesse, G. Anelli, P. Jarron, et al. Three adjacent channels were sent to an Agilent 54832B $1 \mathrm{GHz}$ oscilloscope which displayed each at $16 \times 10^{9}$ 8-bit samples per second, with the oscilloscope triggered by the signal on the central channel.

One hundred events were taken, with one being inadvertently stored twice, providing 99 for study. Each event stored $3 \times 800$ 3 -digit pulse heights separated by $62.5 \mathrm{ps}$, so covering a timespan of $50 \mathrm{~ns}$. 


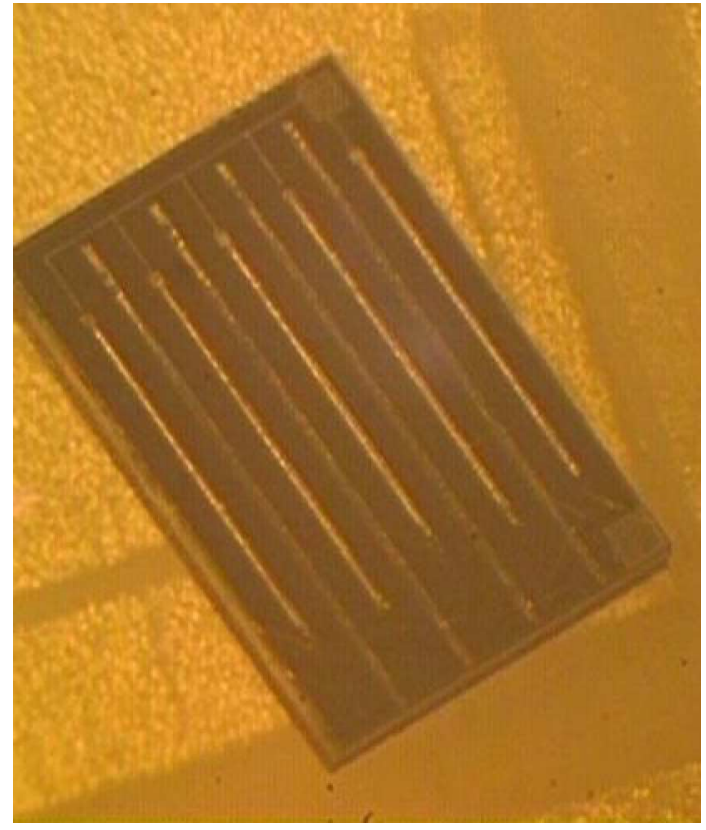

Fig. 6. 3D trench-electrode sensor.
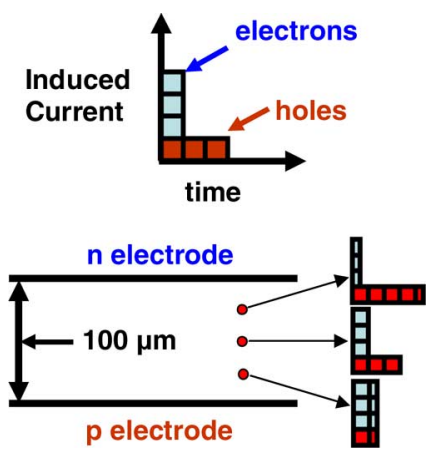

Fig. 7. Schematic diagram of induced currents from a track in a parallel-plate trench-electrode sensor. It is assumed the electron and hole drift velocities are constant with a 3 to 1 ratio. Schematic pulses for three different track paths parallel to the electrode planes and perpendicular to the plane of the diagram are shown in the bottom diagram.

For several reasons, this sensor will not provide the fastest available speeds:

- with 20 ganged cells, the capacitance is on the high side by pixel standards;

- the bias voltage used was well below the maximum possible;

- the operating temperature, $20^{\circ} \mathrm{C}$, is higher than the expected $-20^{\circ} \mathrm{C}$ or lower for operation at the LHC, resulting in lower drift velocities and a slower readout circuit.

In addition, angled tracks that stayed in one channel's electrodes and did not deposit ionization in the two adjacent channels may have a different collection-time behavior from that of normally-incident beam particles.

Fig. 9 shows typical events with pulses in more than one channel. 67 events had a pulse in only one channel. One of them, the 50th to trigger, with a normal shape, had a second pulse in a neighboring channel, but delayed by $4 \mathrm{~ns}$ and with a shape that
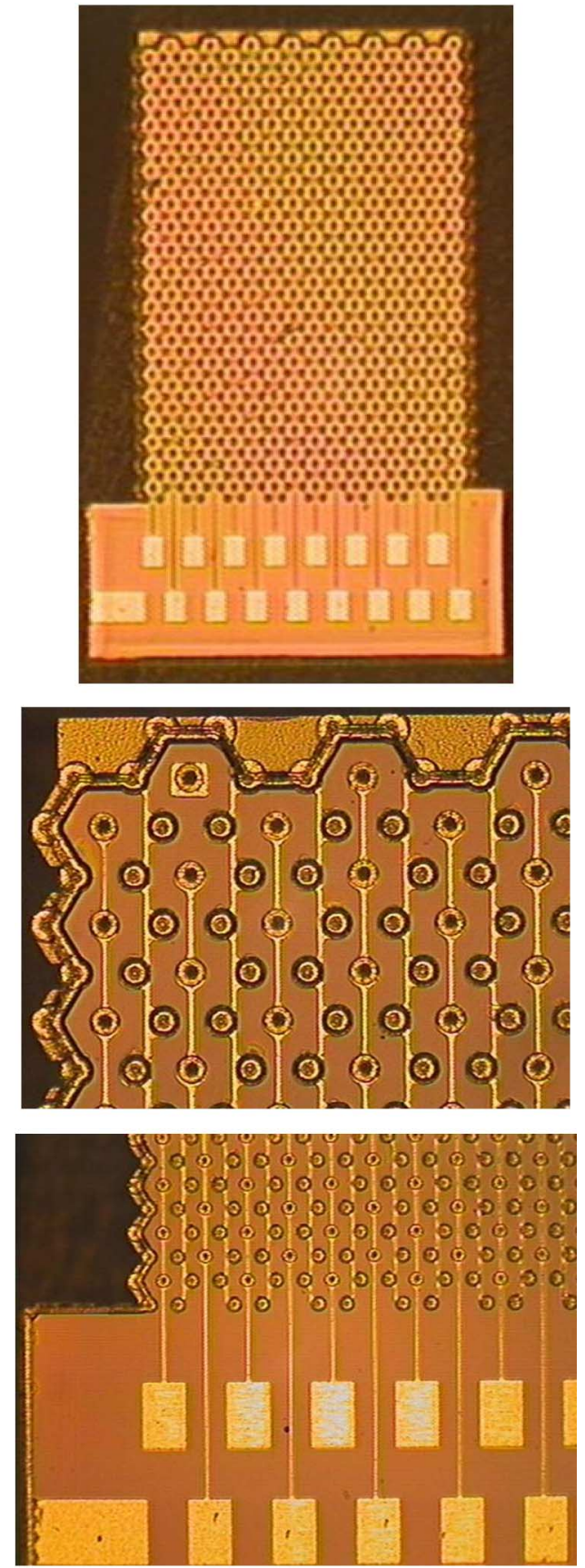

Fig. 8. (top) 3D hexagon-cell active edge sensor tiled with 16 columns, each with 20 hexagons with sides of $50 \mu \mathrm{m}$ connected to the 16 pads at the bottom. (middle) Magnified view of the top corner of the sensor. The dicing etch follows the trench etch route on the left side in the figure, but not on the top. (bottom) Output pad end of 3D active-edge hexagon sensor.

differs from that of the other events. It may have been a combination of noise and the induced signal seen on the non-triggering traces in Fig. 10 which show the first, middle, and last of the 67 events.

Fig. 11 shows the event with the lowest pulse height of the 67 and also the pulse with the largest expected timing error. That pulse is small, but also the phase of the noise has reduced the slope at the $50 \%$ point used here to simulate a constant fraction discriminator set for what is normally the steepest part of the rise. This method is subject to limited statistics. Many such 


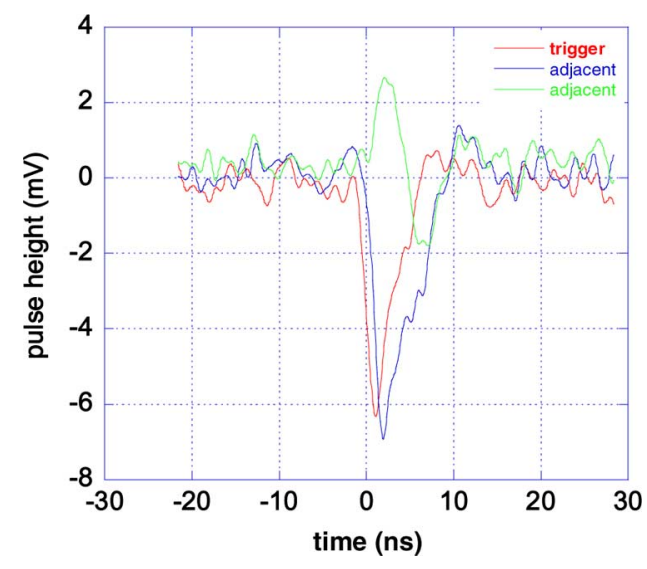

Fig. 9. Event 9 which has tracks in two adjacent columns of pixels and so on two channels. The third trace shows a bipolar pulse induced by capacitative coupling from the adjacent traces. The data for all figures were taken at $20^{\circ} \mathrm{C}$. The underlying points in all traces were separated by $62.5 \mathrm{ps}$. The triggering pulse in all figures is the one closest to zero time.

events would need to be examined to determine the actual error. Two such methods will be described in Sections IX and X for doing that with our limited data.

The noise was measured from the differences between each of the leading 270 points before the start of each pulse and each base line average. Fig. 12 shows a distribution which is Gaussian with no visible non-Gaussian tails. The $\sigma_{\text {noise }}$ is 0.333 $\mathrm{mV}$, and for the $67 \times 270=18090$ points directly, is 0.322 $\mathrm{mV}$. Changing the bin size from 0.1 to $0.05 \mathrm{mV}$ in the plot (not shown) changes the $\sigma$ from $0.333 \mathrm{mV}$ to $0.322 \mathrm{mV}$. The differences between these values are small and within the expected statistical errors. The distribution of these base line averages is also shown in Fig. 12.

While Gaussian, the noise is not white, due to frequency limitations of the amplifier. Its discrete Fourier transform is shown in Fig. 13 (the plotted coefficients are a sum over the sampled noise values rather than an integral, and the continuous $\exp (\mathrm{i} \omega \mathrm{t})$ becomes a discrete phase factor for each term). The distribution, shown from 0 to $1000 \mathrm{MHz}$, peaks at the first of the 16 points $(62.5 \mathrm{MHz})$, drops to $30 \%$ of the peak at $200 \mathrm{MHz}$ and to nearly to zero at $600 \mathrm{MHz}$, corresponding to cycle times of $16 \mathrm{~ns}, 5 \mathrm{~ns}$, and $1.6 \mathrm{~ns}$. These are comparable to time durations for base line full-widths of the 3D sensors: 5-9 ns. Both are limited by the frequency response of the amplifier although the noise does have some low-amplitude pulses of somewhat higher frequency.

\section{Calculation of Noise-Induced Timing ERrors}

Three methods were used to calculate timing errors, $d t$, from noise.

One used the measured rise slopes of each of the 67 events at the $50 \%$ level where they are steepest. Dividing the measured $\sigma_{\text {noise }}$ from the pre-pulse base lines by each slope gives an estimated timing error for that event. This method, while making direct and simple use of the data is, as mentioned earlier, is subject to fluctuations due to the limited available statistics.

A second method summed the six largest pulses which are largely noise-free due to their amplitudes. The cancellation of the noise in the sum further reduces the noise relative to the
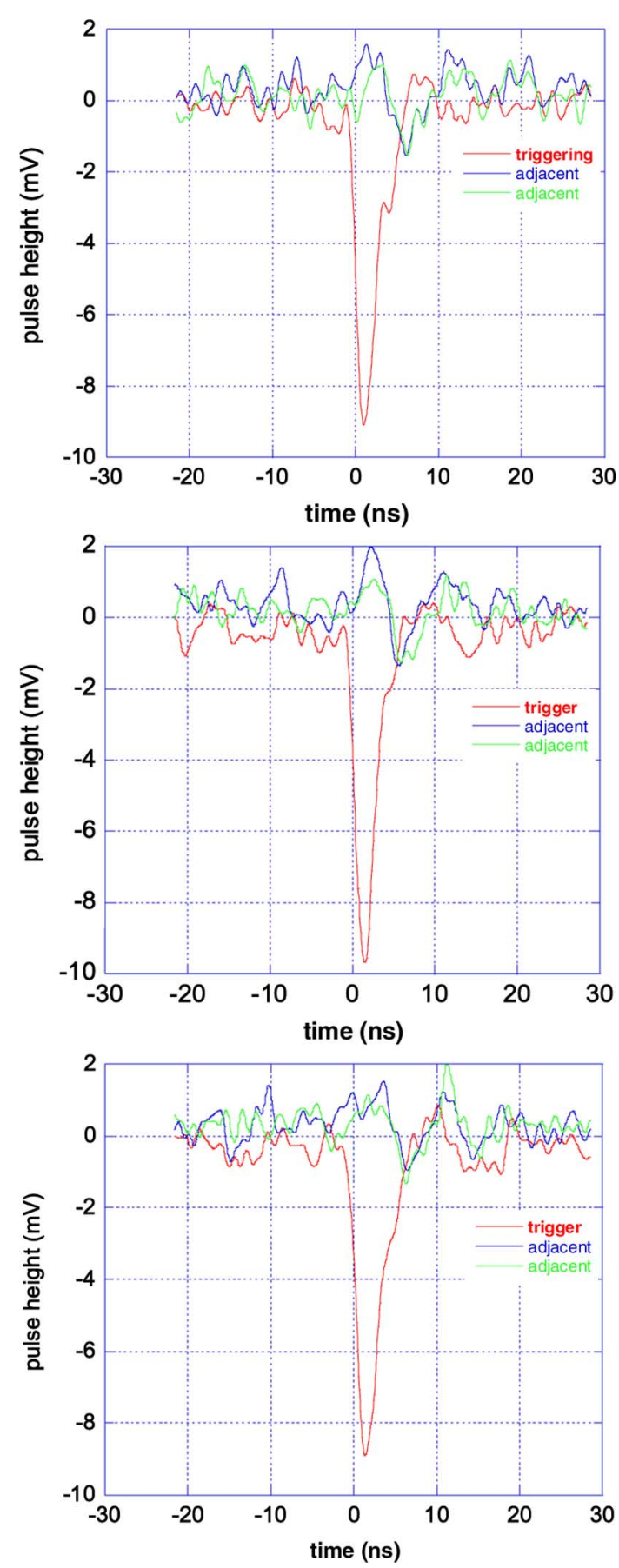

Fig. 10. (top) The first of 67 single-channel events (of 99 total ${ }^{90} \mathrm{Sr}$ triggers). (middle) Event 51, the middle event. (bttom) Event 99, the last ${ }^{90} \mathrm{Sr}$ event.

signal. The average of each pre-pulse trace was subtracted to remove common-mode noise and each trace was subdivided into 3 parts giving 201 noise sequences. The noise-free pulse was scaled to the size of the smallest data pulses, added to the 201 noise sequences, and the first method above was used to give an estimated timing error distribution. This was repeated for a range of pulse amplitudes.

The third method used the pulses from the second method and found the time of the best fit of a noise-free pulse, scaled to the peak amplitude of the pulse with noise (including its noise). 

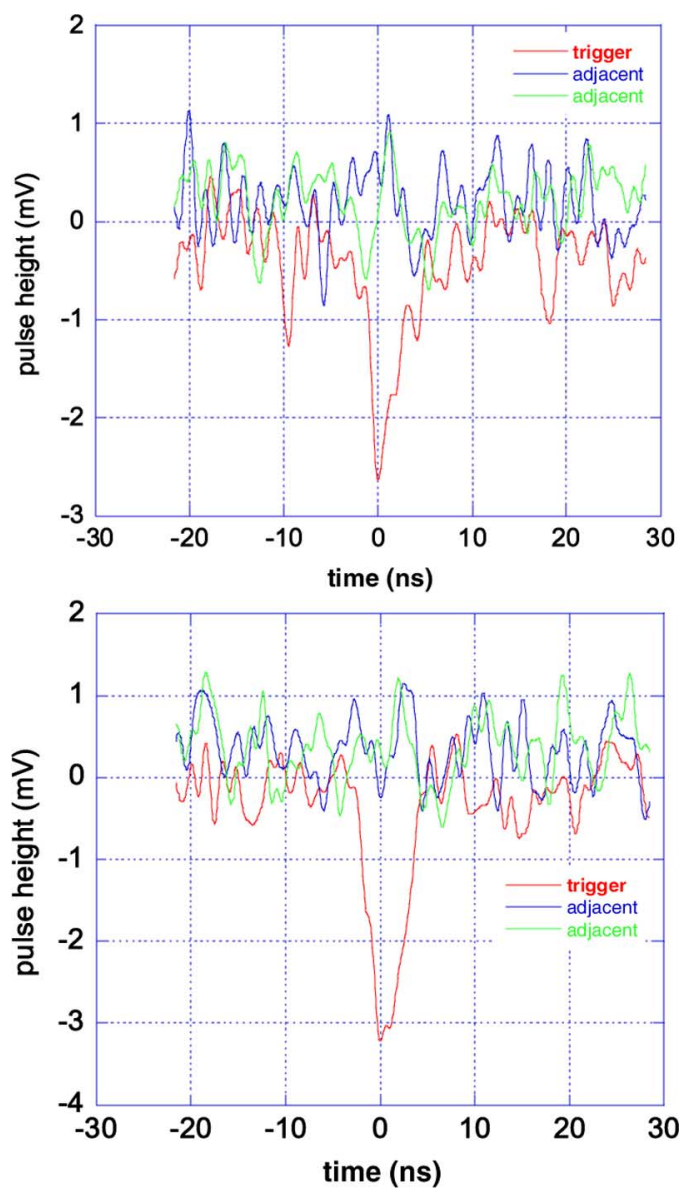

Fig. 11. (top) Event 41, the single-column event with the lowest peak amplitude. (bottom) The single-column event with the largest expected timing error in the central scatter plot of Fig. 14

This was repeated for each pulse amplitude, for the 201 pulses with noise.

\section{Detailed Steps for Calculation}

We can now calculate the expected noise-induced timing error:

1) remove events with pulses in either of the neighboring channels;

2) average the first 270 points to find the base line level;

3) find the peak height (with our negative pulses, the lowest value);

4) find the points closest to $50 \%$, where the slope is greatest, and from them;

5) calculate the slope, $S=\left\{V\left(t_{+}\right)-V\left(t_{-}\right)\right\} /\left(t_{+}-t_{-}\right)$;

6) calculate the noise-induced expected timing error $d t=$ $\sigma_{\text {noise }} / S$.

Fig. 14 shows a scatter plot of $d t$ vs. pulse height and on the top and right-side axes, the projected distributions of each.

The median and average $d t$ values are 129 and 155 ps and there is a clear pulse-height dependence.

Two additional methods, less subject to such errors were used next. Both used data to provide almost pure noise and almost pure noise-free pulses. Steps 1-4 are the same for both.

1. An approximately noise-free signal pulse shape was found by adding the six pulses above $10 \mathrm{mV}$, which
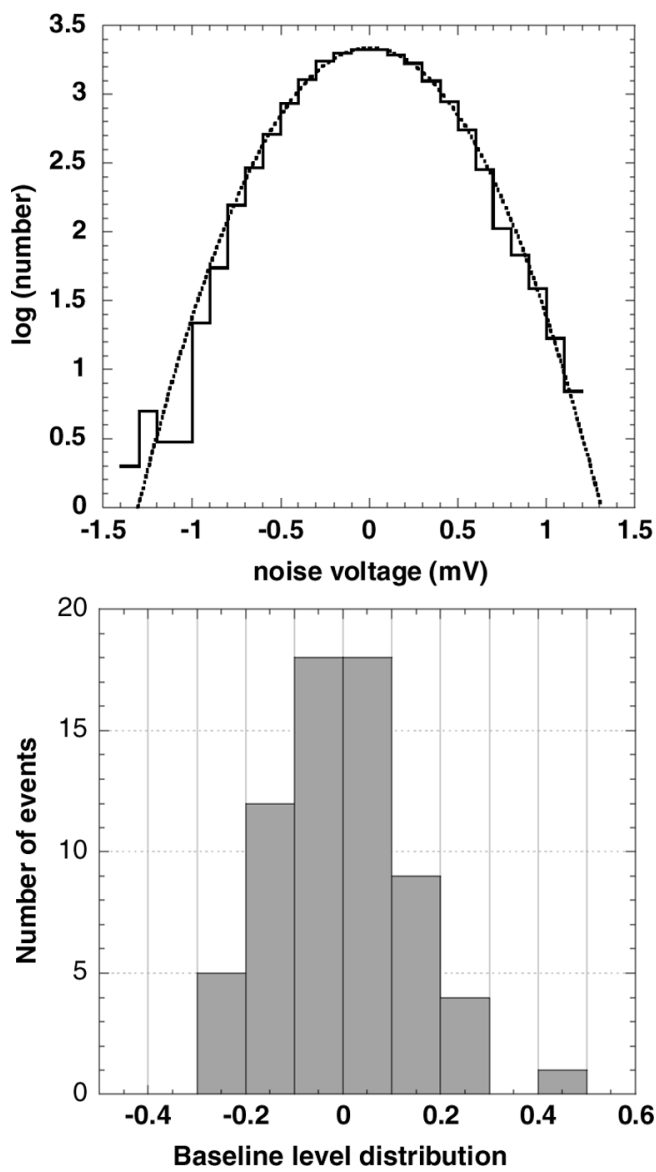

Fig. 12. (top) Log of the fluctuations, relative to the average value, of the 270 points in the base line prior to each of the Sr-90 pulses. The $\sigma$ of the distribution is $0.333 \mathrm{mV}$. (bottom) Event-to-event shift distribution of the entire base line before the pulse. The standard deviation of the fluctuation of 67 base lines is $0.14 \mathrm{mV}$. The range is shifted $+0.246 \mathrm{mV}$ to center on zero.

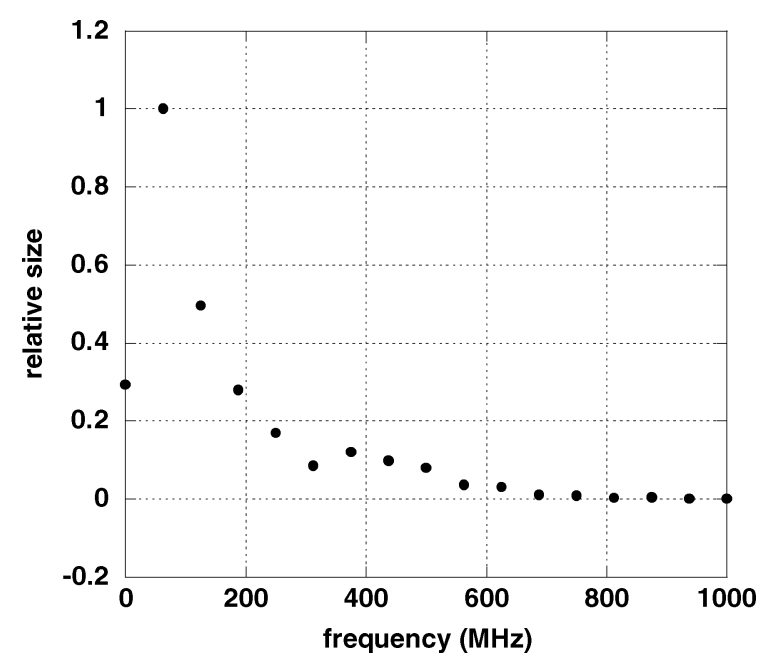

Fig. 13. Fourier transform of the noise.

are already relatively noise-free. To allow for the slight trigger-time variations, the individual curves were shifted by amounts of up to $\pm 0.25 \mathrm{~ns}$ to align the peaks. The noise, being random, tended to cancel as can be seen in Fig. 15, showing the entire sum pulse and Fig. 16, showing with an expanded scale, the leading edge. This noise 


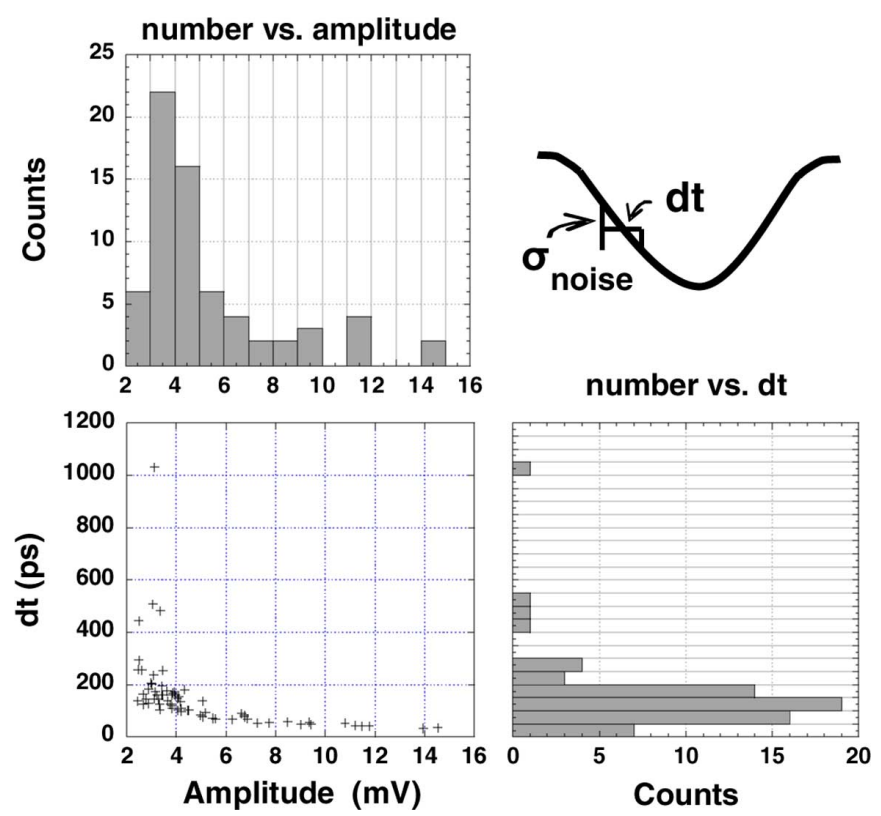

Fig. 14. Scatter plot of expected noise-induced timing errors, $d t$, vs. pulse amplitude, $V$, for 67 pulses and the projections of the $d t$ (right) and $V$ (top) distributions. The projections above and to the right have the same scales as the corresponding axes of the scatter plot The relationship between $d t$, the pulse slope, and $\sigma_{\text {noise }}$ is shown in the upper right diagram. The signal-to-noise ratio is 3 times the pulse height in $\mathrm{mV}$.

cancellation can also be seen in Fig. 17, which shows a pulse from a pulse generator set to have a rise time of $800 \mathrm{ps}$, and also the sum of 5 such pulses. A set of noise sequences was prepared by subtracting the average of each 270-point pre-pulse base line from the 270 points to remove common-mode signals from each of the 67 traces. 2. The 67 baselines were subdivided into $67 \times 3=201$ sets of 90 points each, covering ( 90 points/16 points per $\mathrm{ns})=5.625 \mathrm{~ns}$, a time longer than the pulse-sections to be used in the fit: the rise, once above the noise-level, the top, and the first part of the trailing edge.

3. The stored signal pulse amplitudes were multiplied by a fraction to reduce them to the height of the smallest of the 67 signals.

4. The first noise sequence was added, point-by-point, to the reduced-amplitude signal.

5. Interpolating, the time of the $50 \%$ level-crossing was found.

6. This was repeated for the rest of the $67 \times 3$ noise sets. The signal was next increased in amplitude to that of the second smallest of the 67 , and steps 5 and 6 above were repeated.

7. The $d t$ for each signal amplitude was found from the rms variation about the mean of the $20150 \%$ crossing times and is given in Fig. 18.

This method will be sensitive to the sort of random noise fluctuations seen in Fig. 11.

Finally, the effect of noise was measured by a pulse fitting method. The almost noise-free pulse of step 1 above and the same pulse with the 201 noise sequences of step 3 added, one at a time, were compared. The early, low-level parts of the signal where noise would be most important and the end where the

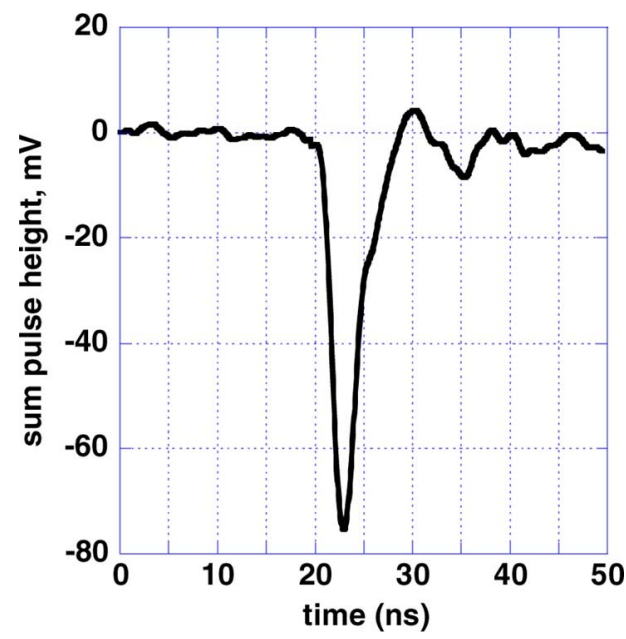

Fig. 15. Sum of the six largest pulses giving an approximately noise-free shape, as the noise starts relatively small compared to each pulse and is then partially cancelled. The full width at half max is $3.0 \mathrm{~ns}$, and is $8.3 \mathrm{~ns}$ at the base.

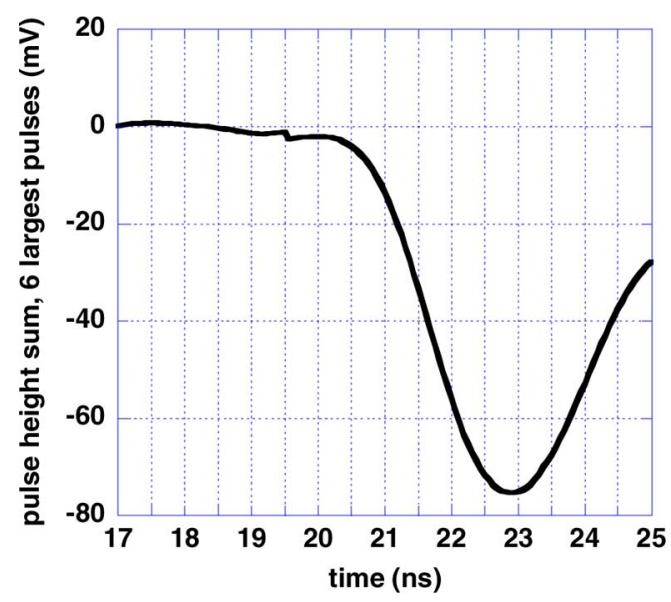

Fig. 16. Leading edge of the sum pulse in Fig. 15.

hole-collection bulge could cause variation were not used. The 62 points used ran from 16 ( $1 \mathrm{~ns})$ to $77(4.8125 \mathrm{~ns})$ from the start of the pulse. Noise was added to those 62 points.

A shorter, more central group of 43 noise-free points from 23 $(1.4375 \mathrm{~ns})$ to $65(4.0625 \mathrm{~ns})$ served as the fitting function and was shifted one point $(62.5 \mathrm{ps})$ at a time, from the front ends matching (23 and 16) to the back ends matching (65 and 77) for a total of $62-43+1=20$ trials. At each position, the 43 differences were calculated. In detail:

8. Following steps $1-4$ and skipping steps $5-7$, the peak of the digital pulse plus noise in step 4 above was used to adjust the peak height of the noise-free fitting pulse and proportionately, all of the other noise-free points, since in real life one does not know the true noise-free pulse height. So all of these points will be off by a common but realistic error factor. Since the same function is used for both the noise-free and noise-added pulses, errors from track angle variations will not be present, but they will also not be present in the first possible use which would employ highenergy, normally-incident tracks.

9. The noise-free amplitudes were subtracted, point-bypoint from the signal plus noise. 

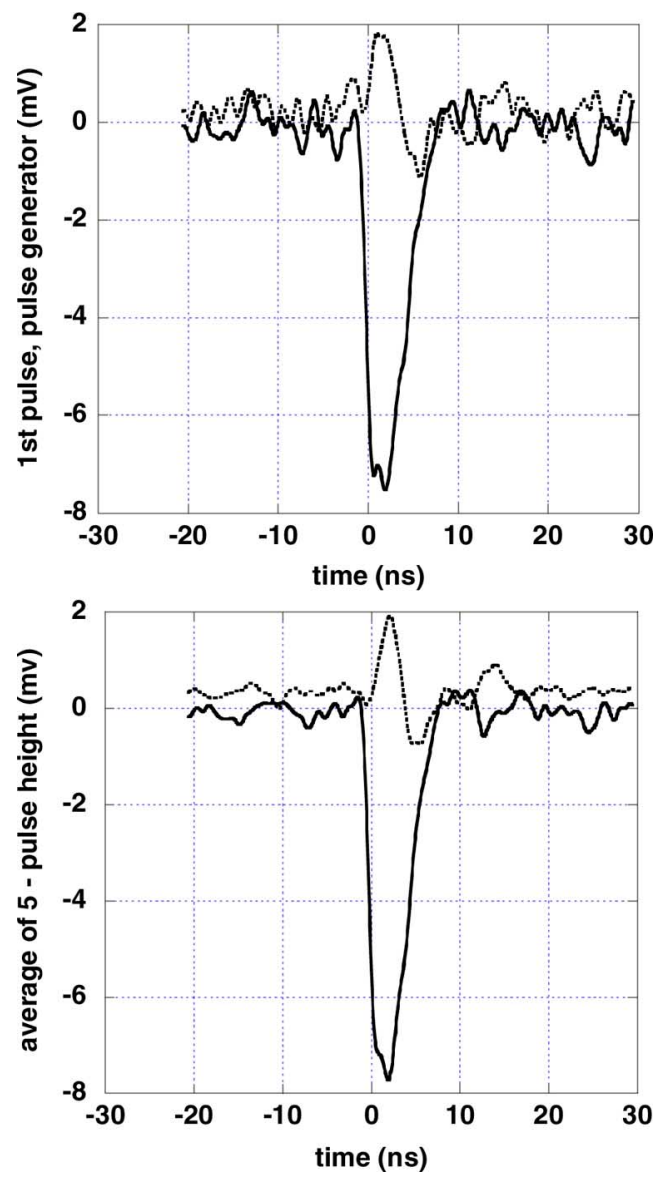

Fig. 17. Pulses from an $800 \mathrm{ps}$ rise-time pulse generator with a neighboring channel (top), and the average of 5 such pulses together with the average of all 10 neighbor-channel pulses (bottom). In contrast to the sensor pulse in Fig. 15, the pulse generator shapes shows no bulge on the trailing edge, indicating the tail on the sensor pulses is not electronic in origin, but rather due to hole motion. It can also be seen that the signals in the neighboring channels have shapes consistent with coupling through inter-channel capacitance, and that the noise of the average pulse is reduced.

10. The standard deviation of these differences was calculated.

11. Steps 9 and 10 were repeated with the same noise-free set shifted one point ( $62.5 \mathrm{ps})$ later.

12. Steps 9 and 10 were repeated for the 19 additional trials and a total shift of $18 \times 62.5 \mathrm{ps}$.

13. The minimum standard deviation for the 20 trials was found.

14. A parabola was fit to that minimum value and the two values on each side. The parabola minimum location will be used to interpolate between the steps. A parabola with $(\mathrm{x}, \mathrm{y})$ points $-x, 0, x$ and $y_{1}, y_{2}, y_{3}(x=62.5 \mathrm{ps})$ has a minimum at

$$
x_{0}=\frac{x\left(y_{1}-y_{3}\right)}{2\left(y_{1}-2 y_{2}+y_{3}\right)} .
$$

15. The standard deviation of these 201 interpolated parabola minima was found and is plotted in Fig. 18.

16. These steps were repeated for a range of pulse heights.

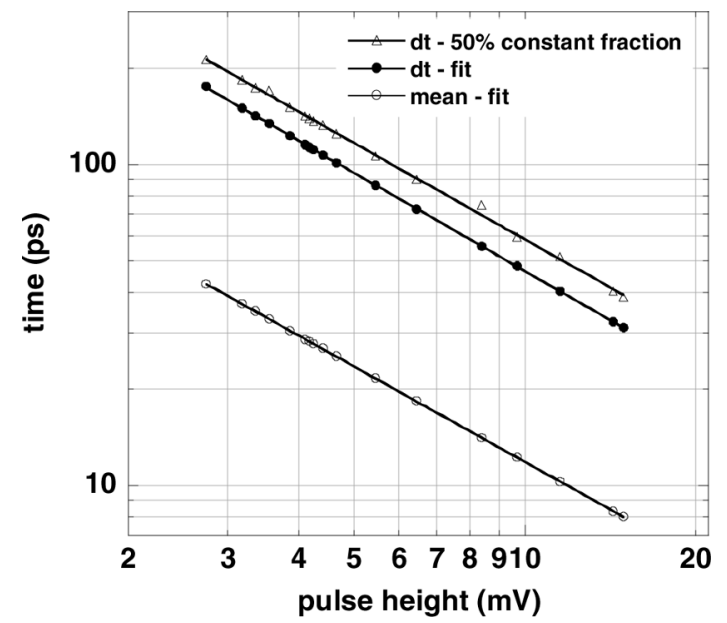

Fig. 18. Expected time errors, dt, due to noise as a function of pulse height from the combined signal pulse shape added to 201 noise segments with dt determined from the standard deviation of time variation of the $50 \%$ point on the leading edge $(\Delta)$ and from the time variation of the best fit time of the combined signal pulse shape to the same shape plus noise $(\bullet)$. The $50 \%$ errors are $20 \%$ larger than the fit errors. The mean value of the best fit times $(O)$ is $24 \%$ of the fit values. The signal-to-noise ratio is 3 times the value of the pulse height expressed in $\mathrm{mV}$.

\section{Results of CALCULATIONS}

We can learn a bit more comparing Fig. 15 and Fig. 17, which shows the $800 \mathrm{ps}$ rise time pulses from the pulse generator. The pulse is injected from one of two calibrate inputs, one to even and one to odd numbered channels. Each channel input goes through an individual on-chip 50fF capacitor. Fig. 17 also shows the sum of 5 such pulses. The pulse generator output shown on the oscilloscope has a rise time of $1.5 \mathrm{~ns}$, only slightly faster than the $1.6 \mathrm{~ns}$ of the ${ }^{90} \mathrm{Sr}$ pulses. This suggests that the ${ }^{90} \mathrm{Sr}$ pulses output from the 3D sensors may have rise times significantly faster than the observed 1.6 ns.

The several ns wide bulge starting at $20 \%$ to $40 \%$ of the peak height on the trailing edges of the pulses in Fig. 10 shows up quite clearly in Fig. 15, is presumably due to the slower hole collection, and is not present in Fig. 17, which shows pulsegenerator pulses. The decrease of noise in Fig. 15 also shows up in Fig. 17 and the capacitative coupling of the pulse to the neighboring channels, which is visible in some of the earlier figures, is quite clear here.

The variation of the noise-induced timing errors, $d t$, with pulse height is shown in Fig. 18. The result is

$$
d t=a / V^{b}
$$

with $a=581.4,485.3,115.2$ and $b=0.9971,1.0171,0.98655$ for the constant fraction method, the fitting method, and the mean of the fitting method. $d t$ is in ps, $V$ is in $\mathrm{mV}$, and the Pearson coefficient is between 0.9993 and 0.99999 .

The timing error is $20 \%$ greater for the constant fraction method; the fitting method is not thrown off by a noise fluctuation at just one specific region (the pulse half-height in this case). The exponents are within $2 \%$ of a $45^{\circ}$ slope, giving 
$d t \approx$ const. $/ V$. This could be used when signals from multiple, sequential sensors are available, to give more weight to the low-noise-error, large pulses (having delta rays), so long as (1) the track is not one of the few that are very close to an electrode and (2) the last part of the pulse is not used for the fit-places where delta rays could cause pulse-shape errors. (These requirements are generally never satisfied by planar sensors.)

Since we know the zero time of the pulse before the addition of the noise sequences, we know the true time and might expect the mean value of the $201 \mathrm{fit}$ times to cluster about zero. Instead we find the mean value of the 201 values at each pulse height to be positive (late) with the non-zero $a$ above. This is due, in the parabolic interpolation of step 12 above, to (1) the use of more points ahead of the peak than after (to avoid the need to simulate the fitting of the tail from hole motion such as the one seen in Fig. 15), and (2) the more gentle slope after the peak. This combination causes a shift of the fitting pulse from the optimum time to one step early to tend to produce a larger error than from one step late, and the parabola responds by generally picking later times.

While the fitting method could be adjusted for this effect, it would be an artificial one. After all, this data is just for a preliminary trial, to examine the effects of noise. The measurements used

- a large-capacitance sensor

- a beta source with the only collimation being the absence of a pulse in adjacent channels

- and employed no real knowledge of the track location. One should note that the fitting pulse shape used here matched the pulse shape to which noise was added. The two pulses will differ somewhat in real life, and a decrease in slope and an eventual leveling off at some fraction of the pulse width is expected, because even in the absence of noise, there will be no unique time where all pulses will agree. Beyond designing for the shortest pulse shape possible where amplitude and width combine to improve resolution, it would be best to combine timing detectors with good tracking, predict the expected pulse shape, including the tail from holes and fit the entire pulse. And this brings us to:

\section{NEXT}

Before examining improvements to silicon technology we should examine the possibility of using diamond sensors. They should provide a modest improvement in performance.

One figure of merit is the charge generated per unit track length times the saturation velocity which gives the input current. Silicon, with more charge but a lower saturation velocity provides a net $35 \%$ more current for equal track lengths.

Ionization charge motion in either sensor causes a redistribution of charge in the signal electrode conductors which will change the voltage at the input transistor gate (but by an amount that is very small compared to the bias voltage, leaving the drift velocity unchanged). Diamond, with its lower dielectric constant, will allow more of that shifted charge for the gate. That voltage change $V_{\text {in }}$ times $A$, the amplifier gain appearing inverted at the output puts a total voltage change $(A+1) V_{\text {in }}$ across the feedback resistor, $R_{\text {feedback }}$. This produces the much lower input impedance $R_{\text {feedback }} /(A+1)$ that appears in parallel with impedance of the input capacitance. This could happen a bit sooner with diamond than silicon by an amount that depends on amplifier, interconnect and sensor details. Although this is not likely to be a major factor, still with their low leakage currents, diamond detectors might provide a useful advantage, but one limited by the smaller industrial base for diamond, the greater cost, and other possible difficulties such as ones that might arise from the more than factor of two difference in coefficients of thermal expansion with a diamond pixel sensor and its readout chip. The improvements below apply the both silicon and to diamond.

There are many potential sources of major gains which are partially listed below. None of them have been used in this paper other than the intrinsic speed of 3D sensors, the fast current amplifier, and use of a waveform recorder (the oscilloscope). One item below, lowering capacitance by increasing the electrode separation of adjacent channels (the horizontal gaps in Fig. 5), will, however, decrease the ratio of average drift to peak electric field and the constancy of the Ramo weighting field. Most of the others can be used simultaneously, given the conditions listed for each:

- use of trench-electrode sensors such as shown in Figs. 5 and 6 , and reducing as far as practicable, their capacitance. Going from the hex-electrode sensors to the trench-electrode ones of Fig. 5 with $2 \times 100 \mu \mathrm{m}$-gaps and a length (in the vertical direction of the figure) of $250 \mu \mathrm{m}$ should, for a slower but otherwise similar $0.25 \mu \mathrm{m}$-technology current amplifier, give an expected factor of 2 decrease in noise and increase in time resolution [44],

- operation at reduced temperatures, giving less noise and greatly increased amplifier and drift speeds [31], [40],

- use of an amplifier with the lowest possible noise, given the available space, heat removal capabilities, and speed requirements,

- use of higher electric fields giving drift velocities close to saturation values (the sensor used for this paper could take double the voltage used),

- use of waveform recorders if a channel can fit within the area of a pixel. Only the large-amplitude part of the signal is needed. One possibility is to determine the baseline by keeping track of its average as a single, updated number in storage.

- use of multiple timing layers of detectors, if allowed by Coulomb scattering, space, and cost considerations—-some possibly rotated to help with tracking,

- use of a weighting factor, as suggested in Section XI, to favor layers having high signal-to-noise ratios,

- and, with similar limitations, use of high-resolution position-tracking layers. (The present amplifier, including the output buffer easily would fit inside a $100 \mu \mathrm{m} \times 200 \mu \mathrm{m}$ pixel, but the input stage, which is all that would be needed before a comparator or wave-form recorder, uses $2.1 \mathrm{~mW}$, as it is optimized for a $5 \mathrm{pF}$ source capacitance. Optimized for a $0.5 \mathrm{pF}$ pixel source, $0.2 \mathrm{~mW}$ should be possible, 
giving $1 \mathrm{~W} / \mathrm{cm}^{2}$, still several times larger than desirable if the other circuit elements operate at the same power density and with limited access to external cooling. In that case, both power considerations for an always-on circuit and space for the following elements indicate that fast-timing pixels would be larger than tracking-only layers.) Thus,

- The most accurate timing will be done by a system, not by one sensor-readout unit.

\section{CONCLUSION}

Expected resolution times have been examined for a detector system using 3D trench-electrode sensors, fast current amplifiers and constant-fraction discriminators or wave-form recorders. Data from a 3D hex-electrode sensor, a fast current amplifier, and a fast oscilloscope indicate front-end amplifier noise sets the present expected limits to shorter time resolution. For that system it ranges from 177 ps for small pulses from a ${ }^{90} \mathrm{Sr}$ beta source to $31 \mathrm{ps}$ for the largest. Several suggested factor-of-two improvements are listed which, used together, could make further significant reductions in these times.

\section{REFERENCES}

[1] E. Heijne, "Muon flux measurement with silicon detectors in the CERN neutrino beams," in Proc. CERN-83-06, 1983, p. 18.

[2] K. Borer, A. G. Clark, R. Engelmann, O. Gildemeister, C. Gössling, E. H. M. Heijne, P. Jarron, B. Lisowski, T. Pal, M. A. Parker, and N. Redaelli, "Construction and performance of a $1 \mathrm{~m}^{2}$ silicon detector in UA2," Nucl. Instrum. Methods Phys. Res. A, Accel. Spectrom. Detect. Assoc. Equip., pp. 548-557, 1987.

[3] J. England, B. Hyams, L. Hubbeling, J. Vermeulen, and P. Weilhammer, "Capacitative charge division read-out with a silicon strip detector," Nucl. Instrum. Methods., vol. 185, pp. 43-47, 1981.

[4] J. Walker, S. I. Parker, B. Hyams, and S. Shapiro, "Development of high density readout for silicon strip detectors," Nucl. Instrum. Meth., vol. 226, pp. 200-203, 1984.

[5] C. Adolphsen, A. Litke, A. Schwarz, M. Turala, G. Anzivino, R. Horisberger, L. Hubbeling, B. Hyams, A. Breakstone, R. Cence, S. I. Parker, and J. Walker, "Initial beam test results from a silicon-strip detector with VLSI readout," IEEE Trans. Nucl. Sci., vol. NS-33, pp. 57-59, Feb. 1986.

[6] R. Sonnenblick et al., "Electrostatic simulations for the design of silicon strip detectors and front-end electronics," Nucl. Instrum. Methods Phys. Res. A, Accel. Spectrom. Detect. Assoc. Equip., vol. A 310, p. 189, 1991.

[7] S. I. Parker, C. J. Kenney, and J. Segal, "3D-A proposed new architecture for solid-state radiation detectors," Nucl. Instrum. Methods Phys. Res. A, Accel. Spectrom. Detect. Assoc. Equip., vol. A 395, pp. 328-343, 1997.

[8] C. Kenney, S. Parker, J. Segal, and C. Storment, "Comparison of 3D and planar silicon detectors," in Proc. 9th Meeting Div. Particles and Fields Amer. Phys. Soc., Minneapolis, MN, Aug. 11-15, 1996, vol. V2, pp. 1342-1345.

[9] C. Kenney, S. Parker, J. Segal, and C. Storment, "Silicon detectors with 3-D electrode arrays: Fabrication and initial test results," IEEE Trans. Nucl. Sci., vol. 46, no. 4, pt. III, pp. 1224-1236, Aug. 1999

[10] C. Kenney, S. Parker, B. Krieger, B. Ludewigt, T. Dubbs, and H. Sadrozinski, "Observation of beta and $\mathrm{X}$ rays with 3D-architecture, silicon micro-strip sensors," IEEE Trans. Nucl. Sci., vol. 48, no. 2, pp. 189-193, Apr. 2001.

[11] S. I. Parker and C. J. Kenney, "Performance of 3-D architecture, silicon sensors after intense proton irradiation," IEEE Trans. Nucl. Sci., vol. 48, no. 5, pp. 1629-1638, Oct. 2001.

[12] C. J. Kenney, S. I. Parker, and E. Walckiers, "Results from 3D sensors with wall electrodes: Near-cell-edge sensitivity measurements as a preview of active-edge sensors," IEEE Trans. Nucl. Sci, vol. 48, no. 6, pt. III, pp. 2405-2410, Dec. 2001.

[13] J. Morse, C. Kenney, E. Westbrook, I. Naday, and S. Parker, "3dx: Micromachined silicon crystallographic x-ray detector," in Proc. SPIE, 2002, vol. 4784, pp. 365-374.
[14] C. Da Via, G. Anelli, J. Hasi, P. Jarron, C. Kenney, A. Kok, S. Parker, E. Perozziello, and S. J. Watts, "Advances in silicon detectors for particle tracking in extreme radiation environments," Nucl. Instrum. Methods Phys. Res. A, Accel. Spectrom. Detect. Assoc. Equip., vol. A 509, pp. 86-91, 2003

[15] J. Morse, C. Kenney, E. Westbrook, I. Naday, and S. Parker, "The spatial and energy response of a $3 \mathrm{~d}$ architecture silicon detector measured with a synchrotron X-ray microbeam," Nucl. Instrum. Methods Phys. Res. A, Accel. Spectrom. Detect. Assoc. Equip., vol. A 524, pp. 236-244, 2004.

[16] C. Da Via, J. Hasi, C. Kenney, A. Kok, and S. Parker, "3D silicon detectors - status and applications," Nucl. Instrum. Methods Phys. Res. A, Accel. Spectrom. Detect. Assoc. Equip., vol. A 549, pp. 122-125, 2005.

[17] A. Kok, G. Anelli, C. Da Via, J. Hasi, P. Jarron, C. Kenney, J. Morse, S. Parker, J. Segal, S. Watts, and E. Westbrook, "3D detectors-State of the art," Nucl. Instrum. Methods Phys. Res. A, Accel. Spectrom. Detect. Assoc. Equip., vol. A 560, pp. 127-130, 2006.

[18] C. J. Kenney, J. D. Segal, E. Westbrook, S. Parker, J. Hasi, C. Da Via, S. Watts, and J. Morse, "Active-edge planar radiation sensors," Nucl. Instrum. Methods Phys. Res. A, Accel. Spectrom. Detect. Assoc. Equip., vol. A 565, pp. 272-277, 2006.

[19] S. Parker, C. J. Kenney, D. Gnani, A. C. Thompson, E. Mandelli, G. Meddeler, J. Hasi, J. Morse, and E. M. Westbrook, "3DX: An X-ray pixel array detector with active edges," IEEE Trans. Nucl. Sci., vol. 53, no. 3, pt. III, pp. 1676-1688, Jun. 2006.

[20] C. J. Kenney, J. Hasi, S. Parker, A. C. Thompson, and E. Westbrook, "Use of active-edge silicon detectors as X-ray beam monitors," Nucl. Instrum. Methods Phys. Res. A, Accel. Spectrom. Detect. Assoc. Equip., vol. A 582, pp. 178-181, 2007.

[21] C. Da Via, J. Hasi, C. Kenney, V. Linhart, S. Parker, T. Slavicek, S. J. Watts, P. Bem, T. Horazdovsk, and S. Pospisil, "Radiation hardness properties of full-3D active edge silicon sensors," Nucl. Instrum. Methods Phys. Res. A, Accel. Spectrom. Detect. Assoc. Equip., vol. A 587, pp. 243-249, 2008.

[22] C. Da Via, S. Parker, M. Deile, T.-E. Hansen, J. Hasi, C. Kenney, A. Kok, and S. Watts, "Dual readout-Strip/pixel systems," Nucl. Instrum. Methods Phys. Res. A, Accel. Spectrom. Detect. Assoc. Equip., vol. A 594, p. 7, 2008.

[23] S. Parker, C. Da Via, M. Deile, T.-E. Hansen, J. Hasi, C. Kenney, A. Kok, and S. Watts, "Dual readout 3D direct/induced-signals pixel systems," Nucl. Instrum. Methods Phys. Res. A, Accel. Spectrom. Detect. Assoc. Equip., vol. A 594, p. 332, 2008.

[24] M. Mathes, M. Cristinziani, C. Da Vià, M. Garcia-Sciveres, K. Einsweiler, J. Hasi, C. Kenney, S. Parker, L. Reuen, M. Ruspa, J. Velthuis, S. Watts, and N. Wermes, "Test beam characterization of 3-D silicon pixel detectors," IEEE Trans. Nucl. Sci., vol. 55, no. 6, pt. II, p. 3731, 2008.

[25] C. Da Vià et al., "3D active edge silicon detector tests with $120 \mathrm{GeV}$ muons," IEEE Trans. Nucl. Sci., vol. 56, no. 2, p. 505, Apr. 2009.

[26] C. Da Vià, E. Bolle, K. Einsweiler, M. Garcia-Sciveres, J. Hasi, C. Kenney, V. Linhart, S. Parker, S. Pospisil, O. Rohne, T. Slavicek, S. Watts, and N. Wermes, "3D active edge silicon sensors with different electrode configurations: Radiation hardness and noise performance," Nucl. Instrum. Methods Phys. Res. A, Accel. Spectrom. Detect. Assoc. Equip., vol. A 604, pp. 505-511, 2009.

[27] C. Fleta, D. Pennicard, R. Bates, C. Parkes, G. Pellegrini, M. Lozano, V. Wright, M. Boscardin, G. F. Dalla Betta, C. Piemonte, A. Pozza, S. Ronchin, and N. Zorzi, "Simulation and test of novel 3D silicon radiation detectors," Nucl. Instrum. Methods Phys. Res. A, Accel. Spectrom. Detect. Assoc. Equip., vol. 579, pp. 642-647, 2007.

[28] G. Pellegrini, M. Lozano, M. Ullán, R. Bates, C. Fleta, and D. Pennicard, "First double sided 3-D detectors fabricated at CNM-IMB," Nucl. Instrum. Methods Phys. Res. A, Accel. Spectrom. Detect. Assoc. Equip., vol. 592, pp. 38-43, 2008.

[29] A. Zoboli, M. Boscardin, L. Bosisio, G.-F. Dalla Betta, C. Piemonte, S. Ronchin, and N. Zorzi, "Double-sided, double-type-column 3D detectors at FBK: Design, fabrication and technology evaluation," IEEE Trans. Nucl. Sci., vol. 55, no. 5, pp. 2775-2784, Oct. 2008.

[30] P. Jarron, F. Anghinolfi, E. Delagne, W. Dabrowski, and L. Scharfetter, "A transimpedance amplifier using a novel current mode feedback loop," Nucl. Instrum. Methods Phys. Res. A, Accel. Spectrom. Detect. Assoc. Equip., vol. A 377, p. 435, 1996.

[31] G. Anelli, F. Faccio, S. Florian, and P. Jarron, "Noise characterization of a $0.25 \mu \mathrm{m}$ CMOS technology for the LHC experiments," Nucl. Instrum. Methods Phys. Res. A, Accel. Spectrom. Detect. Assoc. Equip., vol. A 457, p. 361, 2001. 
[32] G. Anelli et al., "A high-speed low-noise transimpedance amplifier in a $0.25 \mu \mathrm{m}$ CMOS technology," Nucl. Instrum. Methods Phys. Res. A, Accel. Spectrom. Detect. Assoc. Equip., vol. A 512, p. 117, 2003.

[33] J.-F. Genat, G. Varner, F. Tang, and H. J. Frisch, "Signal processing for pico-second resolution timing measurements," Nucl. Instrum. Methods Phys. Res. A, Accel. Spectrom. Detect. Assoc. Equip. vol. A 607, p. 387, 2009.

[34] G. S. Varner and L. L. Ruckman, "Sub-10 ps monolithic and low-power photodetector readout," Nucl. Instrum. Methods Phys. Res. A, Accel. Spectrom. Detect. Assoc. Equip., vol. A602, p. 438, 2009.

[35] S. Ramo, "Currents induced by electron motion," Proc. IRE vol. 27, pp. 584-585, 1939.

[36] W. Shockley, "Currents to conductors induced by a moving point charge," J. Appl. Phys., vol. 9, p. 635, 1938.

[37] G. Cavalleri, E. Gatti, G. Fabri, and V. Svelto, "Extension of Ramo's theorem as applied to induced charge in semiconductor detectors," Nucl. Instrum. Methods Phys. Res. A, Accel. Spectrom. Detect. Assoc. Equip., vol. 92, p. I37, 1971.
[38] Particle Data Group, Particle Physics Booklet pp. 217-221, Jul. 2008.

[39] A. R. Couture, D. Drouin, and R. Gauvin, "CASINO V2.42-A fast and easy-to-use modeling tool for scanning electron microscopy and microanalysis users," J. Scanning, vol. 29, no. 3, pp. 92-101, 2007.

[40] C. Jacoboni, C. Canali, G. Otiaviani, and A. A. Quaranta, "A review of some charge transport properties of silicon," Solid-State Electron., vol. 20, pp. 77-89, 1977.

[41] F. Nava, C. Canali, and L. Reggiani, "On the diffusivity of holes in silicon," J. Appl. Phys., vol. 50, p. 922, 1979.

[42] S. O. Rice, "Mathematical analysis of random noise, Part III: Statistical properties of random noise currents," Bell Syst. Tech. J., vol. 24, p. 55, 1945.

[43] Particle Data Group, Particle Physics Booklet p. 290, Jul. 2008.

[44] M. Despeisse, CERN-THESIS-2006-021, 2006/03/06, p. 103, Fig. 3.12 .

[45] C. Amsler et al., Review of Particle Physics 2008. 